\title{
Implications of Possible Alternate Scenarios for Short and Long Duration GRBs
}

\author{
C. Sivaram ${ }^{1}$, Kenath Arun ${ }^{2, *}$ and Kiren O.V. ${ }^{2}$ \\ ${ }^{1}$ Indian Institute of Astrophysics, Bangalore, 560 034, India \\ ${ }^{2}$ Christ Junior College, Bangalore, 560 029, India
}

\begin{abstract}
Gamma-ray bursts (GRB) are the most luminous physical phenomena in the universe, consisting of flashes of gamma rays that last from seconds to hours. In this paper we look at possible alternate scenarios for both short and long duration GRBs. We consider scenarios for short duration GRBs, which could explainwhy fewer GRBs are observed than what is expected. Also a new class of objects (dark matter objects) is proposed as possible candidate for short duration GRBs which eliminates the baryon load problem, which could also provide a possible scenario for the formation of substellar black holes, distinct from the usual Hawking black hole.We also consider a new model (dubbed the Smashnova model)where an SN can trigger a GRB.In case of long duration GRBs, we propose the possibility of a GRB triggering the collapse of a WR or RG star in a binary system producing an SN, and typical signatures. We also discuss GRBs from population III stars of $\sim 500 M_{\odot}$ at high redshifts,and argue that collapse of such high mass stars does not lead to GRBs. Observational implications for the above scenarios are briefly explained.
\end{abstract}

Keywords: Baryon load problem, Compactness problem, Long duration GRB's, population III stars, Short duration GRB's, Supernova,

\section{INTRODUCTION}

Gamma-Ray Bursts are the most powerful kind of explosion [1]. Their duration can last from a few milliseconds to several hundreds of seconds [2] and based on their duration, they are categorized into 2 classes. Further studies (e.g. ref. [3]), lead to the understanding of the different nature of the progenitor, i.e., a binary system of neutron stars [4] for the short duration GRBs and a collapsar [5] for the long duration GRBs.

In this paper, we look at the possible alternate scenarios for short and long duration GRBs. A new class of objects made up entirely of dark matter particles is proposed as possible candidate for short duration GRB which eliminates the baryon load problem. These could also provide a possible scenario for the formation of sub-stellar black holes, distinct from the usual Hawking black hole. In the scenario for short duration GRBs we consider the possibility of release of neutrinos preceding the gamma ray emission, possibly explaining why fewer GRBs are observed than what is expected. We also consider the case of a WD detonation following break up of a neutron star which by accreting the debris can trigger a short duration GRB. This is a model where an SN can trigger a GRB (dubbed the Smashnova model).

In case of long duration GRBs the initial burst is usually followed by a longer-lived afterglow emitted at longer wavelengths. Here, we propose the possibility of a GRB

*Address correspondence to this author at the Christ Junior College, Bangalore, 560 029, India; Tel: +91-80-4012 9292; Fax: +91-80- 4012 9222;

E-mail: kenath.arun@cjc.christcollege.edu triggering the collapse of a WR or an RG star, in a binary system producing an $\mathrm{SN}$, and typical signatures.

We also discuss GRBs from population III stars of $\sim 500$ $M_{\odot}$ at high redshifts. We argue that collapse of such high mass stars does not lead to GRB as their core collapse temperatures are not sufficient to produce gamma rays.

\section{SHORT DURATION GRB AND DARK MATTER OBJECTS}

Short gamma-ray bursts are those GRB that have a shorter duration $(<0.2-2 \mathrm{~s})$ and a harder spectrum as compared to the duration of 2-200s for long GRB. Short GRBs are possibly due to the merger of two neutron stars. The spectrum observed is harder because the objects merging to produce the GRBs are more compact. In the case of the short duration GRB, the energy released is the binding energy of the neutron stars which is of the order $\sim 10^{53} \mathrm{ergs}$.

\subsection{Baryon Load Problem}

Most sources capable of impulsively releasing the $10^{53} \mathrm{ergs}$ or more of energy required to power a GRB, however, contain so much matter around them that if the energy released were used to accelerate even a very small fraction $\left(\sim 10^{-3}\right)$ of the baryons present, only a non-relativistic wind would result. This is known as the baryonloading problem $[6,7]$. 
Table 1. Primordial black hole mass with dark matter particle mass.

\begin{tabular}{|c|c|}
\hline $\boldsymbol{m}_{\boldsymbol{D}}(\mathrm{GeV})$ & $\boldsymbol{M}_{\boldsymbol{D}}(\mathrm{g})$ \\
\hline \hline 10 & $10^{29}$ \\
\hline 100 & $10^{27}$ \\
\hline 250 & $4 \times 10^{25}$ \\
\hline 500 & $10^{25}$ \\
\hline 1000 & $3 \times 10^{24}$ \\
\hline
\end{tabular}

There is hope, to the effect that the geometry of the sources is such, that at least some of the energy released is channelled along directions relatively free of baryons, so that relativistic bulk motion and the ensuing beaming of radiation may occur along certain lines of sight. So far, this has not yet been fully demonstrated for any theoretical source of GRBs [8-10].

\subsection{Dark Matter Particle Objects}

Here, we discuss a new class of objects made of pure dark matter (DM) particles. If these dark matter particles (of mass $m_{D} \sim 10 \mathrm{GeV}$ to $1 \mathrm{TeV}$ ) cluster and form gravitationally bound objects, these pairs of dark matter particles can annihilate throughout these objects. These dark matter particleantiparticle pairs can undergo annihilation and produce high energy gamma rays which could be detected. These high energy gamma rays could be a signature of this new class of objects [11].

The Chandrasekhar mass (upper limit) for these degenerate DM objects is given by:

$$
M_{D(C H)}=\left(\frac{\hbar c}{G}\right)^{3 / 2} \frac{1}{m_{D}^{2}}
$$

For a dark matter particle of mass $m_{D} \sim 100 \mathrm{GeV}$, this works out to be:

$$
M_{D} \approx 10^{27} g=10^{-6} M_{\text {sun }}
$$

The size of these objects is given by (for the usual degenerate gas configuration; thermal energy not being relevant):

$M_{D}^{1 / 3} R=\frac{92 \hbar^{2}}{G m_{D}^{8 / 3}}$

For the $10^{-6}$ solar mass object the size works out to be:

$R \approx 10^{5} \mathrm{~cm}$

If their mass exceeds this limit, they will collapse to form black holes of size given by:

$R_{S}=\frac{2 G M}{c^{2}} \approx 1 \mathrm{~cm}$
(For DM particles of mass $m_{D}=100 \mathrm{GeV}$ )

The energy released during the collapse is given by:

$E=\frac{G M^{2}}{R} \approx 10^{48} \operatorname{ergs}$

This energy is released in the form of gravitational waves.

If equal amount of baryonic matter collapses along with the dark matter to form the black hole, then the baryonic matter will be heated up to a temperature $T$, according to:

$$
M R_{g} T=10^{48} \text { ergs }
$$

This gives $T \approx 10^{12} K, R_{g}$ being the universal gas constant.

This energy corresponds to gamma ray frequencies. Since the mass heated up is $\sim 10^{-6}$ solar mass, in this scenario, the 'Baryon Load' problem seems ameliorated as the relativistic kinetic energy corresponds to Lorentz factor of $\sim 10^{2}-10^{3}$.

The time scale of the gamma-ray burst here is given by:

$t_{\text {burst }}=\sqrt{\frac{R^{3}}{G M}} \approx 0.01 \mathrm{~s}$

The matter will expand to $>10^{9} \mathrm{~m}$ in a few seconds. Depending on the ambient medium there could be an afterglow. The expansion could cause lowering of the temperature, resulting in production of X-rays, UV, etc, that is, radiation of successively longer wavelengths over longer intervals of time. The peak wavelength would scale with the expansion time scale as roughly $\lambda \sim t^{-1}$, so that a few days after the initial burst, the wavelength would be in the ultraviolet to visible but with intensity far less (by a factor of $10^{4}$ ) than the initial luminosity.

As is the case here, magnetic fields are not expected to be present [11], the radiation would not be polarised like in some GRB sources. This could be an alternative scenario for short duration $(0.1-0.01 s)$ sub-luminous gamma-ray bursts.

Again in this scenario, unlike in some other models of short duration GRB's we do expect much lower flux of neutrinos and gravitational waves to be simultaneously emitted (for details see ref. [11]). This could be another distinct signature of this model.

\subsection{Alternate Scenario for formation of Primordial Black Holes}

As an additional consequence this could be another way in which primordial black holes $(\mathrm{PBH})(<$ stellar mass $)$ can form, apart from Hawking black holes. The masses of these sub-stellar mass black holes depend on the mass of the dark matter particles given by equation (1). For different dark 
matter particle masses the black hole mass is given in the Table 1: (see also ref. [11])

These PBH will then evaporate due to the usual Hawking radiation with a life time given by:

$$
\tau_{e v}=\frac{5120 \pi G^{2} M^{3}}{\hbar c^{4}}
$$

For this lifetime to be of the order of the Hubble time, the mass of the black hole should be:

$$
M \approx 10^{14} g
$$

This implies that we would expect all of the above black hole masses (formed by the collapse of DM dominated objects) to be still present at the present epoch of the universe.

\section{SMASHNOVA}

In recent papers $[12,13]$ a new model was proposed (Smashnova model), where a supernova is followed by a gamma-ray burst. For a very close binary system, the white dwarf (close to Chandrasekhar mass limit) can detonate due to tidal heating, leading to a supernova. Material falling on to the neutron star at relativistic velocities can cause its collapse to a magnetar or quark star or black hole leading to a gamma-ray burst.

If a white dwarf impacts a red giant (RG), it would take about 2 months to penetrate the bloated RG. The RG would collapse, becoming another WD. If the white dwarfs merge, it can form a neutron star. This will release about $10^{53}$ ergs of binding energy. NS impacting a RG or red supergiant (SG) can cause an SN outburst first followed by collapse of a NS and the in-falling material into a black hole and finally leading to GRB. An NS colliding with a WR star will result in $\mathrm{SN}$ followed by GRB, as the core collapses to a BH.

Black holes in a certain mass range can tidally disrupt a neutron star [14], leading to a $10^{53} \mathrm{ergs}$ GRB. In the case of a WD and an NS close binary, the WD can be tidally stretched or broken up when the separation is about $R_{W D}$. CO white dwarf (close to $N_{c h}$ ) can detonate due to heating.

Tidal energy of the order of $10^{50} \mathrm{ergs}$ can heat WD to about $10^{9} \mathrm{~K}$.

This is enough to detonate $\mathrm{C}$ and this can hence lead to an SN. Enough material falls on an NS at velocities greater than about 10 percent the speed of light. About $5 \times 10^{32} \mathrm{~g}$ of matter falling in has a kinetic energy of $\sim 10^{52} \mathrm{ergs}$. On impact, gamma rays of nuclei energy $\geq 1 \mathrm{MeV}$ is released with more than $10^{52}$ ergs in $\gamma$ - ray photons.

Neutron stars can be spun up and the flux squeezing can increase the magnetic field. When the NS slows down due to dipole radiation (Magnetar), in-falling matter can make it collapse to a $\mathrm{BH}$ releasing more than $10^{53}$ ergs, with the acceleration of particles due to the magnetic field. Tidal stretching and heating can considerably increase thermonuclear (detonation) rates, especially carbon burning. The recent calculations are discussed in detail in ref [13].

\section{GAMMA-LESS GAMMA-RAY BURSTS}

Observations of an afterglow of the GRBs reveal that the source energies (released at large distances $(\sim \mathrm{Gpc})$ ) of well over $10^{44} \mathrm{~J}$ are released in gamma rays [15]. The relativistic

energies acquired by the ejected material help resolve what was earlier known as the compactness problem. The optical depth of the source (with $F$ being the observed fluence in gamma rays) is given by:

$$
\tau=\frac{f_{P h o t} \sigma_{T} F D^{2}}{m_{e} c^{2} R_{S}^{2}}
$$

For duration $\Delta t$ of the burst, the source size $R_{S} \leq c \Delta t$, $\sigma_{T}$ is the Thompson cross section, $D$ is the distance of the burst and $f_{\text {Phot }}$ is the fraction of photons which can produce gamma rays in the pair annihilation process: $\gamma \leftrightarrow e^{-}+e^{+}$.

For typical values ( $F=10^{-6} \mathrm{ergs} / \mathrm{cm}^{2}, D \approx 1 G p c$, $\Delta t \sim 1 s$ ), typical optical depths are very huge, that is, $\tau \approx 10^{15}-10^{16}$ ! So gamma rays cannot escape from this region, i.e. from the area around the central engine (the compactness problem) [16]. The resolution of the problem lies in considering the Lorentz factors associated with the relativistic bulk motion $(\gamma)$. The modified formula for the optical depth is: [17]

$$
\tau=\frac{f_{\text {Phot }} \sigma_{T} F D^{2}}{\gamma^{2 \alpha} m_{e} c^{2}\left(\gamma^{2} c \Delta t\right)^{2}}=\frac{f_{\text {Phot }} \sigma_{T} F D^{2}}{\gamma^{4+2 \alpha} m_{e} c^{2}(c \Delta t)^{2}}
$$

The above picture, applies to GRBs where sufficient energy is converted to accelerate the ejected matter to relativistic speeds. It is also commonly thought that this may be essentially the difference between supernovae and GRBs, in the sense, that the latter involve matter ejected at relativistic energies (the total rest energy corresponding to that of the collapse of a massive star, perhaps a WR star). There have been associations of GRBs with type Ic SN, whose progenitors are WR stars.

For short duration GRBs, the popular model is merger of two compact objects, i.e. neutron stars (NS) or tidal break up of an NS by a black hole. It is possible, that in some situations, the matter is not accelerated to relativistic speeds and the gamma rays are indeed trapped inside the region. In this case, the optical depth is very high (as given by equation (11)). 
However, in this case, as the temperatures could be $\sim 10^{10} \mathrm{~K}$ (corresponding to $2 m_{e} c^{2} / k_{B}$ ), we have the neutrino pair annihilation process dominating: [18]

$\gamma+\gamma \rightarrow e^{+}+e^{-} \rightarrow v+\bar{v}$

The reaction rate and energy released goes as $T^{9}$, where $T$ is the temperature. Essentially, the energy released per unit volume is given by:

$\varepsilon \sim n_{e^{+}} n_{e^{-}} \sigma_{W} V k_{B} T$

Both $n_{e^{+}}$and $n_{e^{-}}$are proportional to $T^{3}, \sigma_{W}$, the weak cross section is taken as $T^{2}\left(E^{2}\right)$ and the energy of each neutrino is $\sim k_{B} T$. So $\varepsilon$ scales as $T^{9}$.

$\varepsilon \propto T^{9}$

Indeed we can write:

$\varepsilon \sim \frac{G_{F}^{2} E^{2}}{(\hbar c)^{4}} n_{e^{+}} n_{e^{-}} k_{B} T$

Where $G_{F}=1.5 \times 10^{-49} \mathrm{ergcm}^{3}$ is the universal Fermi weak interaction constant:

$$
\begin{gathered}
n_{e^{+}} \approx n_{e^{-}} \sim\left(\frac{k_{B} T}{\hbar c}\right)^{3} \\
\left(E \sim k_{B} T\right)
\end{gathered}
$$

This gives $\varepsilon \approx 10^{20} \mathrm{ergs} / \mathrm{cm}^{3}$.

For a volume $(c \Delta t)^{3}$, where $\Delta t \sim 1 s$, this implies that $10^{44} \mathrm{~J}$ of neutrino energy is produced in a second. So about $10^{58}$ neutrinos are released (with average energy of several $\mathrm{MeV})$.

This implies that we could have many gamma-ray bursts where no gamma rays are produced, but only neutrinos. With the neutrino cross section, $\sigma_{W}$, equation (11) gives,

$$
\tau \approx 0.1
$$

So the neutrinos should be able to freely escape. There is no associated compactness problem in this case! Furthermore, there could be many gamma-ray bursts which do not produce gamma rays, but only high energy neutrinos!

Neutrino flux from GRBs is too weak to be detected at present (at Gpc distances) and hence cannot at present be compared with observations. We only point out that there are possibly fewer bursts than expected because of this possible scenario of energy going into neutrinos. For a GRB 1Gpc away, the flux is $10^{-8}$ times that of SN1987A. To detect 10 events, the detector will need $10^{12}$ Tonnes of water. This is beyond any current neutrino technology. It may also be noted, even the optical flux, even for a $30 \mathrm{~m}$ telescope, from a distance of $1 \mathrm{Gpc}$ is only 3 photons/day.

If the source of the central engine is merger of two neutron stars (like for the popular model of short duration GRBs), then most of the binding energy is anyway expected to be released through neutrinos $\left(\sim 10^{53} \mathrm{ergs}\right)$ in a duration of few seconds. As neutrinos couple weakly (to matter) the radiation force may not accelerate the ejected material to relativistic speeds. The conversion $v+\bar{v} \rightarrow \gamma+\gamma$ can take place, which would fall off with distance $R$, from the central source as $1 / R^{8}$, so that a 'pressure gradient' is set up.

\subsection{Gamma - Neutrino Processes}

Again as the magnetic fields in this picture of the central engines (i.e. neutron star merger) are expected to be large $\left(\sim 10^{12} G\right)$, there could be associated gamma - neutrino processes such as plasma-neutrino losses, especially for neutrinos with a magnetic moment (i.e. $\gamma \rightarrow v \bar{v}$ ). The emission rate density is given by: [19-21]

$$
\dot{E}_{v}=\frac{\mu_{v}^{2} \omega_{0}^{4}}{c^{3}} \int_{\omega_{0}}^{\infty} \frac{\omega\left(\omega^{2}-\omega_{0}^{2}\right) d \omega}{\exp \left(\hbar \omega / k_{B} T\right)-1}
$$

Where, $\mu_{v}$ is the neutrino magnetic moment and $\omega_{0}$ is the plasma frequency.

The neutrino Bremsstrahlung processes scale as $\sim T^{6}$. The net result shows that the above processes lower the gamma ray flux and enhance the neutrino flux considerably. Upper limit on the neutrino luminosity of such sources from phase-space considerations have been given in ref. [22].

Now as the neutrinos drain away energy from the source region, the optical depth (equation (11)) could drop steeply (even without relativistic motion!) and gamma ray emission could subsequently follow but with much reduced intensity. The afterglow would now correspond more to that of a typical SN. Considering the wide variety of possible phenomena, scenarios such as the one above could also be kept in mind while discussing extremely energetic events.

This may explain (apart from beaming factor) why fewer gamma-ray bursts are seen than what is expected. However, in the case of neutron star mergers, gravitational waves would be detectable, as it is independent of the optical depth. So the signature of such gammaless GRB's could be simultaneous detection of neutrinos and gravitational waves.

\section{LONG DURATION GRB'S AND GRB'S TRIGGER- ING SUPERNOVA EXPLOSION}

The initial burst of a GRB is usually followed by a longer-lived afterglow, which is emitted at longer wavelengths. 
The evidence for a physical connection between GRB and core collapse supernovae (SN) has increased since the discovery of GRB afterglows [23, 24]. So far SN signatures have been found in only a few GRB.

It is generally believed that the long-duration gamma-ray bursts are associated with the deaths of massive stars in a kind of supernova-like event such as a hypernova [25]. Here we propose that a GRB in a binary system with a progenitor of SN, such as a Wolf-Rayet (WR) star or massive red giant star (RG) could trigger the collapse of the WR or RG star producing a supernova explosion [26]. A paradigm for the correlation between GRBs and supernovaebased on the data from the Chandra (iron emission lines in the afterglow of GRB 991216) are discussed in [27, 28]which further support the theory that links the origin of the energy of GRB's to the extractable energy of electromagnetic black holes, leading to the GRB-supernova correlation.

The gamma-ray energy released in a typical GRB is of the order of $\sim 10^{52}$ ergs. The flux of gamma rays at a distance of about $50 \mathrm{AU}$ is:

$f=\frac{E_{G R B}}{4 \pi D^{2}} \approx 10^{21} \mathrm{ergs} / \mathrm{cm}^{2} / \mathrm{s}$

And the corresponding energy density is given by:

$\varepsilon=\frac{f}{4 c} \approx 10^{10} \mathrm{ergs} / \mathrm{cc}$

\subsection{Gamma-Ray Burst and Red Giants}

The total gamma-ray energy falling on the RG (or WR) in a duration of several seconds will be far more (trillion times) than the bolometric radiation emitted by the star. Hence this flux of gamma rays can induce the collapse of the RG star to produce an SN. On the RG the energy falling $\sim 10^{50}$ ergs (on a WR star it is $\sim 10^{45}$ ergs).

For a red supergiant, like Betelgeuse, the gravitational pressure $\left(\approx \frac{G M^{2}}{R^{4}}\right) \sim 1$ dyne $/ \mathrm{cm}^{2}$.

The isotropic gamma-ray flux from the GRB falling on the red supergiant would be $10^{10}$ times more, or if it is focused, would be $10^{13}$ times larger! If the source of gamma rays is collimated into a beam at an opening angle of $\sim 5^{0}$, then the flux received by the companion star will be more by a factor of $\sim 10^{3}$ and the energy received will be focused in an area of $\sim 10^{28} \mathrm{~cm}^{2}$.

The time taken for the collapse is given by:

$\tau=\left(\frac{R^{3}}{G M}\right)^{1 / 2} \sim 100$ days

for RG and a few days in the case of WR star.

This could possibly explain the lag in observing the associated SN signature after the GRB [29-31].
In case the companion star is another WR star of similar mass, metallicity and rotation, there is a possibility that it may also collapse into a black hole giving rise to another gamma-ray burst. So a possible signature of such a scenario (i.e. a WR binary, where one of the stars collapses to cause a GRB) is two consecutive GRB's separated by perhaps several days, from the same region of the sky, which at a Gpc distance corresponds to an angular separation of $\left(\sim 10^{15} / 10^{28} \mathrm{rad}\right) \sim 0.02$ microarcsec.

Of course gamma ray resolution is nowhere near this figure, but if there are optical afterglows associated with both explosions and these spread over $10^{15} \mathrm{~m}$, then they would be just within the current interferometric precision of about 20 microarcsec! So we should see two afterglows within 20 microarcsec separated by several weeks $[32,33]$.

Again, if the companion is a red supergiant (SG), then the resulting core collapse SN would again be spatially separated by a picoarcsec with a time lag of a few months! Perhaps future space telescopes of the ATLAS type or the OWL on the ground could be sensitive to these phenomena.

\subsection{Gamma-Ray Burst and Main Sequence Stars}

If solar like main sequence stars, or white dwarfs(WD) are within few light years from such a GRB (occurring in dense star forming regions) then the gamma ray flux on a MS star is:

$f=\frac{E_{G R B}}{4 \pi D^{2}} \approx 10^{14} \mathrm{ergs} / \mathrm{cm}^{2} / \mathrm{s}$

(Total gamma ray irradiation would be $\sim 10^{37}$ ergs $\sim 10^{3}$ times its bolometric luminosity!)

A white dwarf close to the Chandrasekhar limit at a distance of $\sim 1$ light-year from the GRB would experience a gamma ray irradiation of $\sim 10^{33} \mathrm{ergs}$, heating it to a temperature:

$T=\left(\frac{f}{\sigma}\right)^{1 / 4}>10^{5} K$

It is possible that this would give rise to a nova like outburst. At that distance, this may not generate a collapse of the WD.

A main sequence star at a distance of a few light-years from the GRB would be irradiated with a gamma ray flux of;

$f=\frac{E_{G R B}}{4 \pi D^{2}} \approx 10^{13} \mathrm{ergs} / \mathrm{cm}^{2} / \mathrm{s}$

which could exhibit high energy flares, of several thousand times their bolometric luminosity for several minutes, with a corresponding increase in temperature:

$T=\left(\frac{f}{\sigma}\right)^{1 / 4}>10^{4} K$ 


\subsection{Gamma-Ray Burst and Star Formation}

Although GRBs could have drastic effects on neighbouring stellar objects, they could inhibit continuing star formation, as even an interstellar gas cloud hundred parsecs away could be heated to several thousand degrees and dissipate. For example, a cloud of $10^{2}$ solar mass, with average density of 100 atoms/cc would be in virial equilibrium only at $\mathrm{T} \sim 20 \mathrm{~K}$ and dust grains could be heated and vaporised by the gamma ray flux [34].

\section{HIGH REDSHIFT POPULATION III STARS AND GRB}

It is now believed that the long-duration gamma-ray bursts (> 2s) are associated with the beamed energy from a specific kind of hypernova event, such as the death of supermassive stars once its silicon "burning" is complete with a Zero Age Main Sequence (ZAMS) mass between 40 and 100 $M_{\odot}$ causing a direct collapse of the core to a black hole [16, $26,35]$. The close connection between GRBs and Type Ib/c supernovae shows that the progenitor stars are almost exclusively low metallicity (at ZAMS) fast rotating Wolf-Rayet stars [36, 37].

The thermal energy associated with a black body at temperature $\mathrm{T}$ is given by:

$E=\frac{4}{3} \pi a T^{4} R^{3}$

Where, $R$ is the size of the compact region (close to that for a black hole).

If the size is compact enough at a certain temperature, the gravitational energy, given by $\frac{G M^{2}}{R}$, associated with the dominant black body radiation will also be significant. The total energy, mass energy plus gravitational, is then given by: [38]

$$
\begin{gathered}
E=\frac{4}{3} \pi a T^{4} R^{3}-G\left(\frac{4}{3} \pi a T^{4} / c^{2}\right)^{2} R^{5} \\
\text { (Putting } M=\frac{4}{3} \pi a T^{4} R^{3} \text { ) }
\end{gathered}
$$

As the gamma rays observed correspond to a temperature of $T \sim 10^{12} \mathrm{~K}$, for the compact opaque region, we estimate the size $R$ for which the two quantities in equation (27) become comparable.

This gives;

$$
R=\left(\frac{3}{4 \pi} \frac{c^{4}}{a T^{4} G}\right)^{1 / 2} \approx 200 \mathrm{~km}
$$

as the radius of the compact region, which following the usual scenario for gamma-ray bursts, would correspond to be about the Schwarzschild or gravitational radius. Equation (28) corresponds to a black hole mass of the order of 60-70 $M_{\odot}$ for a required temperature of $\sim 10^{12} \mathrm{~K}$ to produce gamma rays.

This puts an upper limit on the size of stars that can collapse to give a GRB as $R_{S} \propto M$. The upper limit (for $T \sim 10^{12} \mathrm{~K}$ ) of about 60 solar masses corresponds quite well to the mass of the supposed progenitor stars of GRB, i.e. Wolf-Rayet stars, in the usual scenario. The particles in the system will be in thermal equilibrium with the black body radiation. Therefore, similar analysis with gas thermal energy $\left(N k_{B} T\right)$ yields a similar result.

Equation (28) implies the significant result that much larger stellar masses corresponding to the primeval population III stars [39-41] (i.e. 500 to $10^{3} M_{\odot}$ (or greater)), would result in a much smaller temperature, prior to the collapse into a black hole. This would in turn imply that gamma rays could not be produced by collapse of such stars. So future searches for gamma-ray bursts (at higher $\mathrm{z}$ ) would be perhaps constrained, largely by the type of progenitors which could produce such events.

\subsection{Neutrino Pair Production at High Temperatures}

The argument can also be extended to the case of neutrino pair production at high temperatures. As in the case of a typical core collapse of an SN (like SN1987A) most of the gravitational binding energy is expected to be released in the form of neutrinos (and antineutrinos) of all three flavours $[42,43]$.

The core temperature of the 'proto-neutron star' is around $10^{12} \mathrm{~K}$ (it may collapse to a black hole subsequently). For the case of neutrinos, the black body formula for energy density would be: [44].

$$
\varepsilon_{\text {rad }} \approx \frac{7}{16} a T^{4}
$$

For three flavours, it would be:

$$
\varepsilon_{\text {rad }} \approx \frac{7}{16} a T^{4} \times 3 \times 2
$$

This when used in equations (27) and (28) would lead to the radius $\mathrm{R}$ as:

$$
R=\left(\frac{3}{4 \pi} \frac{c^{4}}{\left(\frac{7}{16} a T^{4} \times 3 \times 2\right) G}\right)^{1 / 2} \approx 125 \mathrm{~km}
$$

So this implies for $T \sim 10^{12} \mathrm{~K}$, a mass of $\sim 40 \mathrm{M}_{\odot}$.

This in turn, would constrain the type of progenitor which could produce, simultaneously, both gamma rays and 
neutrinos. Perhaps only short duration bursts, caused by merger of two neutron stars or tidal break up of an NS can lead to such simultaneous intense bursts of both gamma rays and neutrinos [45].

In any case, massive population III stars above $250-300$ $M_{\odot}$ are expected to collapse into black holes [42, 43] and those in the range $140-250 M_{\odot}$ are expected to undergo the so called Pair Instability Super Nova, i.e. PISN, where production of $e^{+} e^{-}$pairs at around the oxygen burning temperature at $2 \times 10^{9} \mathrm{~K}$ would reduce the radiation pressure by $\frac{1}{8} a T^{4}$ and cause a collapse followed by complete fragmentation of the star. Above $250 M_{\odot}$, the collapse terminates in a black hole, which, by the above argument would not produce a gamma-ray burst (or a neutron star).

\section{POSSIBLE HIGH - Z PROGENITORS FOR GRB}

The earliest stellar objects forming in the universe are expected to be very massive, well above hundred solar masses. This is mainly because of a paucity of metals to increase opacity in the collapsing gas clouds. However, in recent scenarios, DM particles could condense (as they decouple much earlier) into gravitating structures [11,39].

It must be kept in mind, that helium constituting onefourth of the baryonic matter in the early universe can decouple earlier than hydrogen, and condense on to the DM cores. This could build up a supermassive star (of the HoyleFowler type) with a million solar masses or more [14]. The core temperature of such a configuration could rise to $3 \times 10^{8} \mathrm{~K}$ and helium nuclei thermonuclear reactions (triple $\alpha$, for example) could be triggered halting the cloud collapse. This object could release $10^{44} \mathrm{ergs} / \mathrm{s}\left(10^{10} \mathrm{~L}_{\odot}\right)$, and last a million years (burning helium at a rate of $10^{25} \mathrm{~g} / \mathrm{s}$ ) before collapsing again.

Hydrogen burning (pp) could also occur in a shell (around the core). Such objects at high z, would be bright IR sources as seen in JWST, TMT, etc. Their collapse would result in a supermassive black hole perhaps accounting for the origin of such objects. In unusual scenarios, for instance, in the merger of intermediate mass black holes (IMBH), such objects take too long to form, though they have been detected at distances of 12 billion light-years.

Pair-instability supernovae, with core masses less than $250 M_{\odot}$, could explosively detonate releasing a total energy of $10^{46} \mathrm{~J}$, at a rate of $10^{40} \mathrm{~W}$. They would rival quasars but the emission would be all shifted to mainly IR. For core masses above $300 M_{\odot}$, black holes form, perhaps IMBH. If their initial angular momentum is sufficiently high, their collapse to the final stage would be delayed (for angular momentum $>10^{55}$ erg.s ) and the energy could be released in relativistic jets [46]. Such a gamma-ray burst would be at least ten times or more energetic than the usual $10^{45} \mathrm{~J}$ energy release, but depending on $\mathrm{z}$, could be shifted to x-ray region with primarily IR afterglows.

Recent discovery of a relic star, SDSS J0018-0939, which has been around for 13 billion years by Aoki et al. [47] with several chemical peculiarities like very low abundance of elements like $\mathrm{C}, \mathrm{Mg}$, Ca relative to Iron, suggests that it formed from a pair-instability SN explosion of a monster primordial star (a few hundred times the solar mass). These detonations occur at O-burning temperatures, converting the debris to mainly iron (which is enhanced). IMBH could form from heavier core masses. Thus, the brightest SN and GRB at high $-\mathrm{z}(>10)$ could release hundred times more energy than their canonical counterparts at lower $\mathrm{z}$. They would appear as intense point IR sources or $\mathrm{X}$-ray sources (for X-ray bursts).

\section{CONCLUSION}

Previously, we have looked at possible scenarios for short duration GRB. Short duration gamma-ray bursts due to collapse of dark matter dominated objects successfully eliminates the 'Baryon Load Problem'. The remnant of these GRB will be sub-stellar mass black holes, and this mechanismalso leads to the formation of primordial black holes, apart from the usual Hawking black holes.

We also propose the possibility of a GRB triggering the collapse of a WR or RG star in a binary system producing an $\mathrm{SN}$, and typical signatures which accounts for the longerlived afterglow emitted at longer wavelengths which usually follows the initial burst of a GRB.

The compactness problem in GRBs have been tackled here by invoking the Lorentz factors associated with the relativistic bulk motion. This could give a scenario where release of neutrinos precedes the gamma ray emission, possibly explaining why fewer GRB's are observed than what is expected.

In the paper, we argue that collapse of high mass stars (population III stars of $\sim 500 M_{\odot}$ at high redshift) does not lead to gamma-ray burst as their core collapse temperatures are not sufficient to produce gamma rays. Hence it is not possible to observe short duration GRB from high redshift, whereas long duration GRB can be detected.

Observationally the gamma ray resolution is nowhere near what is required to detect the signatures suggested here. Finally, we propose possible models which can perhaps be verified by future space telescopes of the ATLAS type or the OWL on the ground.

\section{CONFLICT OF INTEREST}

The author confirms that this article content has no conflict of interest.

\section{ACKNOWLEDGEMENTS}

Declared none. 


\section{REFERENCE}

[1] Meszaros P. Gamma-ray bursts. Rep Prog Phys 2006; 69: 2259

[2] Kouveliotou C, Meegan CA, Fishman GJ, et al. Identification of two classes of gamma-ray bursts. Astrophys J 1993; 413: L101.

[3] Fong W, Berger E. The locations of short gamma-ray bursts as evidence for compact object binary progenitors. Astrophys J 2013; 776: 18

[4] Eichler D, Livio M, Piran T, Schramm DN. Nucleosynthesis, neutrino bursts and gamma-rays from coalescing neutron stars. Nature 1989; 340: 126 .

[5] Woosley SE. Gamma-ray bursts from stellar mass accretion disks around black holes. Astrophys J 1993; 405: 273.

[6] Kluzniak W. Neutrino oscillations and gamma-ray bursts. Astrophys J 1998; 508: L29.

[7] Piran T. Towards understanding gamma-ray bursts. In: Bahcall JN, Ostriker JP, Eds. Unsolved problems in astrophysics. Princeton: Princeton University Press 1997; p. 343.

[8] Rasio FA, Shapiro SL. Hydrodynamical evolution of coalescing binary neutron stars. Astrophys J 1992; 401: 226.

[9] Kluzniak W, Lee WH. Simulations of binary coalescence of a neutron star and a black hole. Astrophys J 1998; 494: L53.

[10] Wilson JR, Salmonson JD, Mathews GJ. A binary neutron star GRB model. In: Meegan CA, Preece RD, Koshut TM, Eds. AIP conference proceedings 4, gamma-ray bursts. New York: AIP 1998; p. 788.

[11] Sivaram C, Arun K. New class of dark matter objects and their detection. Open Astron J 2011; 4: 57.

[12] Sivaram C. Smashnova model. Astrophysics 2007; 1: arXiv:0707.1091.

[13] Sivaram C, Arun K, Kiren OV. Nuclear detonation around compact objects. Phys Int 2014; 5: 36.

[14] Sivaram C. Some constraints on quasar progenitors and quasar evolution. In: Swarup G, Kapahi VK, Eds. Quasars; proceedings of the international astronomical union symposium No. 119. Dordrecht: D. Reidel Publishing Co 1986; p. 417.

[15] Piran T, Granot J. Theory of GRB afterglow. In: Costa E, Frontera F, Hjorth J, Eds. ESO astrophysics symposia, gamma-ray bursts in the afterglow Era. Berlin: Springer Verlag 2001; p. 300.

[16] Piran T. Gamma-ray bursts and the fireball model. Phys Rep 1999; 314: 575.

[17] Giannios D. Powerful GeV emission from a gamma-ray-burst shock wave scattering stellar photons. Astron Astrophys 2008; 488: L55.

[18] Perkins DH. Particle Astrophysics $2^{\text {nd }}$ Edition. Oxford: Oxford University Press 2009.

[19] Sivaram C. Production and acceleration of ultra high energy particles by black holes and strings. In: Shapiro MM, Silberberg R, Wefel JP, Eds. Currents in high-energy astrophysics. Netherlands: Springer 1995; p. 177.

[20] Raffelt GG. Core mass at the helium flash from observations and a new bound on neutrino electromagnetic properties. Astrophys $\mathrm{J}$ 1990; 365: 559 .

[21] Sivaram C. Neutrino emission from supernovae in the presence of magnetic fields. In: Caffo M, Fanti R, Giacomelli G, Renzini A, Eds. Proceedings of the Third ESO-CERN Symposium; Astronomy, cosmology and fundamental physics. Boston: Kluwer Academic Publishers 1989; p. 471.

[22] Sivaram C, Arun K, Samartha CA. Phase-space constraints on neutrino luminosities. Mod PhysLettA 2008; 23: 1470.

[23] vanParadijs J. From gamma-ray bursts to supernovae. Science 1999; 286: 693.

[24] Cobb BE, Bailyn CD, van Dokkum PG, Buxton MM, Bloom JS. The Supernova associated with GRB 031203: SMARTS opticalinfrared light curves from 0.2 to 92 days. Astrophys J 2004; 608: L93.

[25] Piran T. The physics of gamma-ray bursts. Rev Mod Phys 2004; 76: 1143 . and explosions in "failed supernovae". Astrophys J 1999; 524: 262. Ruffini R, Bianco CL, Fraschetti F, Xue SS, Chardonnet P. On a possible gamma-ray burst-supernova time sequence. Astrophys $\mathrm{J}$ 2001; 555: L117.

[28] Ruffini R, Bernardini MG, Bianco CL. In: The role of GRB 031203 in clarifying the astrophysical GRB scenario. Grebenev S, Sunyaev R, Winkler C, Eds. The Obscured Universe. Proceedings of the VI INTEGRAL Workshop. Noordwijk: ESA Publication Division 2007; p. 561

[29] Galama T, Groot PJ, van Paradijs J, et al. The decay of optical emission from the gamma-ray burst GRB970228. Nature 1997; 387: 479 .

[30] Bloom JS, Kulkarni SR, Djorgovski SG. The observed offset distribution of gamma-ray bursts from their host galaxies: a robust clue to the nature of the progenitors. Astron J 2002; 123: 1111.

[31] Gonzalez ME, Kaspi VM, Lyne AG, Pivovaroff MJ. An XMMnewton observation of the high magnetic field radio pulsar PSR B0154+61. Astrophys J 2004; 610: L37.

[32] Djorgovski SG. Kulkarni SR, Bloom JS. The GRB host galaxies and redshifts. In: Costa E, Frontera F, Hjorth J, Eds. ESO astrophysics symposia, gamma-ray bursts in the afterglow era. Berlin: Springer Verlag 2001; p. 218

[33] Berger E, Cowie LL, Kulkarni SR, Frail DA, Aussel H, Barger AJ A Submillimeter and radio survey of gamma-ray burst host galaxies: a glimpse into the future of star formation studies. Astrophys J 2003; 588: 99

[34] Treumann R, Baumjohann W. Advanced space plasma physics. London: Imperial College Press 1997.

[35] Chincarini G, Fiore F, Della Valle MG, et al. Gamma-ray bursts: learning about the birth of black holes and opening new frontiers for cosmology. Messenger 2006; 123: 54

[36] Detmers RG, Langer N, PodsiadlowskiPh, Izzard RG. Gamma-ray bursts from tidally spun-up Wolf-Rayet stars? Astron Astrophys 2008; 484: 831

[37] vanMarle AJ, Langer N, García-Segura G. Constraints on gammaray burst and supernova progenitors through circumstellar absorption lines. Astron Astrophys 2007; 469: 941.

[38] Sivaram C. Energy enigmas in the early universe and related problems in Newtonian cosmology. Astrophys Space Sci 1988; 140: 403.

[39] Sivaram C. A general upper limit on the mass and entropy production of a cluster of supermassive objects. Astrophys Space Sci 1982; 86: 501

[40] Sivaram C. Towards a unification of the parameters underlying elementary particles and cosmology. Astrophys Space Sci 1982; 88: 507.

[41] Sivaram C. Large number coincidences and unification of the parameters underlying elementary particles astrophysics and cosmology. In: de Sabbata V, Karade TM, Eds. Relativistic astrophysics and cosmology; proceedings of the Sir Arthur Eddington centenary Symposium. Singapore: World Scientific 1984; p. 228.

[42] Kreckel H, Bruhns H, Cizek M, et al. Experimental Results for $\mathrm{H}_{2}$ formation from $\mathrm{H}^{-}$and $\mathrm{H}$ and implications for first star Formation. Science 2010; 329: 69.

[43] Heger A, Woosley SE. The nucleosynthetic signature of population III. Astrophys J 2002; 567: 532.

[44] Gal-Yam A, Mazzali P, Ofek EO, et al. Supernova 2007bi as a pair-instability explosion. Nature 2009; 462: 624.

[45] Bromm V. To cool or not to cool. Science 2010; 329: 45.

[46] Sivaram C, Arun K. Intermediate mass black holes: their motion and associated energetics. Adv High Energy Phys 2014; 2014 924848.

[47] Aoki W, Tominaga N, Beers TC, Honda S, Lee YS. A chemical signature of first-generation very massive stars. Science $2014 ; 345$ 912. 